This is the final draft, after peer-review, of a manuscript published in Human-Computer Interaction. Lecture Notes in Computer Science 2014;8512:289-300. The definitive version, detailed above, is available online at www.springerlink.com

\title{
Can a Theory-Informed Interactive Animation Increase Intentions to Engage in Physical Activity in Young People with Asthma?
}

\author{
Jennifer Murray ${ }^{1}$, Brian Williams², Gaylor Hoskins ${ }^{2}$, Silje Skar ${ }^{2}$, John McGhee $^{3}$, \\ Dylan Gauld ${ }^{4}$, Gordon Brown ${ }^{5}$, Shaun Treweek ${ }^{6}$, Falko Sniehotta ${ }^{7}$, Linda Cameron ${ }^{8}$, \\ Aziz Sheikh ${ }^{9}$, and Suzanne Hagen ${ }^{10}$ \\ ${ }^{1}$ Edinburgh Napier University, School of Life, Sport and Social Sciences, Edinburgh, Scotland \\ j.murray2@napier.ac.uk \\ ${ }^{2}$ Nursing, Midwifery and Allied Health Professions Research Unit, University of Stirling, \\ Stirling, Scotland \\ \{brian.williams, gaylor.hoskins, silje.skar\} astir.ac.uk \\ ${ }^{3}$ College of Fine Arts, University of New South Wales, Australia \\ john.mcghee@unsw. edu. au \\ ${ }^{4}$ Duncan of Jordanstone College of Art and Design, University of Dundee, Dundee, Scotland \\ d.gauld@dundee.ac.uk \\ ${ }^{5}$ Asthma UK Scotland, Edinburgh, Scotland \\ gbrowneasthma.co.uk \\ ${ }^{6}$ Health Services Research Unit, University of Aberdeen, Aberdeen, Scotland \\ s.treweek@abdn.ac.uk \\ ${ }^{7}$ Newcastle University, Newcastle, England \\ falko.sniehotta@ncl.ac.uk \\ ${ }^{8}$ University of California Merced, California, USA \\ 1cameron@ucmerced.edu \\ ${ }^{9}$ The University of Edinburgh, Edinburgh, Scotland \\ Aziz.Sheikh@ed.ac.uk \\ ${ }^{10}$ Nursing, Midwifery and Allied Health Professions Research Unit, Glasgow Caledonian \\ University, Glasgow, Scotland \\ s.hagen@gcu.ac.uk
}

\begin{abstract}
A theoretically-informed interactive animation was developed, using themes drawn from psychology, sociology, applied health research, and narrative theory, which aimed to encourage young people with asthma to engage in physical activity. The animation was evaluated using qualitative and quantitative methods. A web-based Interactive Modelling Experiment was used to evaluate whether the animation was effective in three key areas: knowledge about asthma, inhaler use, and intention to increase physical activity. One-to-one interviews and focus groups were used to evaluate the acceptability of the animation and whether the theoretical basis was effective. Preliminary qualitative findings indicate good acceptability and perceived effectiveness. The quantitative findings are less clear, with a change in simulated activity and inhaler use being found, but with no clear association between these changes and the animation itself. Future work will be carried out to established whether these levels
\end{abstract}


of acceptability and perceived effectiveness are actually translated into behaviour change.

Keywords: Asthma, interactive animation, wIME, theory-informed, multidisciplinary.

\section{Introduction}

Visualisation techniques are increasing in popularity as health care interventions [1]. However, despite this increasing popularity, the majority of visual health interventions are not being developed using sound theoretical bases or even explicit theoretical [2] or design frameworks [1]. This lack of definition and explicit targeting of the underlying mechanisms upon which an intervention is based may impede the success of and evaluation of the intervention; only through understanding and defining the concepts and constructs that the intervention seeks to change and embedding these using a solid theoretical framework can the success of an intervention be measured.

In order to move away from inductive, intuitive creation of health promotion visual interventions, a four stage process for development and evaluation has been developed [1], based on the UK Medical research Council Framework for Development and Evaluation of Complex Interventions [3]. The model proposed is a staged process. To begin, a theoretical basis should be established (the conceptual content). Following this, the modelling structure/visual narrative should be developed, with the 'look' of the intervention then being created. An iterative process of development may continue until a suitable solution is reached at this point, moving between the design stages. Following this, a period of modelling checking should occur, to establish the interpretation of the intervention and its potential impact.

The current research sought to evaluate the effectiveness of an interactive animation that had been developed in line with the described framework for developing complex visual interventions for use in health care [1] and that had a strong, explicit theoretical framework informing its development and content [4]. In brief, cognitive learning theories, health behaviour/behaviour change theories, self-efficacy, narrative theories and HCI principals were embedded within the animation in order to attempt to optimise its propensity for intention and behaviour change [4]. The animation itself aims to increase intentions to (and ultimately increase) engagement in physical activity among young people with asthma (aged 12-18 years), and to increase their knowledge about their asthma and safe/proper inhaler use.

In the UK, the incidence of asthma has increased significantly [5], with approximately one in five children in the UK now being affected by asthma [6, 7]. It is known that physical activity levels among healthy young people is dropping in industrialised nations [8], and that young people with asthma are even less likely than their peers to be physically active [9], despite the evidence linking physical activity to improved asthma control/coping [10], reduced hospital admissions, reduced absenteeism from school, fewer consultations with health professionals, and reduced medication use [11]. 
There is therefore an urgent need to develop strategies that address the unique barriers faced by young people with asthma in order to increase physical activity and exercise, and interventions that are tailored to young people specifically. The animation that the current paper will evaluate was developed with this need as a key focus. The hypotheses for the evaluation are:

H1: The animation will increase people's simulated intentions to engage in physical activity.

H2: The animation will increase people's intentions to engage in safe/proper inhaler use.

\section{Method}

\subsection{Design}

Using a web-based Interactive Modelling Experiment (wIME), a 2x2 mixed factorial design was used to evaluate the effectiveness of the animation, with participants completing a 'baseline' online questionnaire and then completing the same questionnaire approximately two weeks later after either being shown the animation or being assigned to a control group (no animation or alternative was shown). The dependent variables were people's responses on the questionnaire. In addition, one-to-one indepth interviews and small focus groups were used to assess the acceptability of the animation and to identify whether the theoretical basis for the animation was acceptable and effective. Framework analysis was used to analyse the qualitative data.

\subsection{Participants}

For the wIME, participants were recruited online, via social networking sites (e.g., Facebook, Twitter) and through specific online asthma-interest groups (e.g., Asthma UK forums). Snowballing was also encouraged, with participants being asked to distribute the link to the questionnaire to potentially interested parties. Target participants were young people with asthma (12-18years) or parents of young people with asthma. Fifty-three participants completed the initial questionnaire (pre-intervention; 24 parents, 29 young people). Twenty-six participants completed both the preintervention and post-intervention questionnaire (14 parents, 12 young people), indicating a $49 \%$ completion rate. Of those who participated in both questionnaires, 16 were allocated to the control condition and 10 to the animation intervention condition. The mean age of the young-people participants was 17.3 years (17.3 years animation condition; 17.2 years control condition). Seven were male and 19 were female (parents answered on behalf of their male/female child, where it was a parent who responded). Participants were randomly allocated to conditions. All participants indicated that they had asthma or were a parent of a young person with asthma.

For the one-to-one interviews, four participants were recruited via the National Health Service in Scotland. Young people with asthma were identified by an asthma nurse and were approached and informed about the study (with their parent) and invited to participate by the health professional. A 'young person' was defined as 
someone aged between 12-18 years, for the purposes of the current research, as this was the age group for which the animation was primarily developed/designed for. Those who were interested were then contacted by the researchers and provided with additional information. All participants were offered the opportunity to have their parents present during the interview. The focus groups consisted of health professionals and school physical education teachers. Participants were recruited through purposeful sampling, by pre-identifying potentially interested parties through mailing lists, asthma interest groups, and through contacting primary clinics directly. Two focus groups were carried out, involving eight participants in total. Interviews and focus groups lasted approximately 40 minutes to one hour in length.

\subsection{Materials}

The animation was developed using theory to guide the content and 'look', using a multi-disciplinary perspective and included theoretical bases drawn from applied health research, HCI, sociology and psychology. A fuller description of the theoretical development for the animation is detailed elsewhere [4]. The animation aimed to increase levels of physical activity in young people with asthma, while also increasing their knowledge about their asthma and what occurs within the lungs when they are breathless during exercise in comparison to being breathless due to their asthma, and their knowledge about inhaler use. In addition to these key areas of outcome measurement, the animation also was embedded with information to promote safetyasthma messages and self-efficacy building messages (theories that were deemed to be important based on the existent literature and previous in-depth qualitative work carried out by the research team in relation to asthma and physical activity). The animation lasts for approximately seven minutes and allows the user to select a male or female character, which type of activity the character will take part in (running, dance or football) and, at various stages, whether the character will take an inhaler or not. After the animation has been played the user has the option to re-run selecting different options to view different outcomes. For the purpose of the evaluation, to allow consistency across user experiences, users were only able to select running as an activity but could still select their character and the inhaler use options. The animation was developed to appeal to 12-18 year olds and emulated the look of a popular 3D animation style used in film. Colours, visual content, textual and speech content were carefully selected by the research team to enhance the learning opportunities and coherence. The animation's development was further informed by an online consultative user group. This group consisted of 23 people; a mixture of young people with asthma, health professionals, parents, and school staff. The group were consulted on key elements of the animation as they were under development (e.g., character design, gender and activity types to be used in the animation, narratives and storyboarding) in order to increase the animation's acceptability and resonance with key stakeholders. This process was found to be highly informative in shaping the final artefact.

The wIME was hosted on LifeGuide (www.lifeguideonline.org). A wIME is a way of carrying out exploratory work prior to conducting a full-scale trial [12]. Key elements of the intervention can be manipulated to simulate real world effects. 
The benefits of IMEs to inform behaviour change research have been demonstrated $[13,14]$, thus the method was deemed a cost effective and suitable first step in evaluating the effectiveness of the animation. The wIME consisted of a large number of questions which fit under various sub-themes, including asthma perceptions, asthma knowledge, asthma severity, questions specific to theoretical bases for the animation (e.g., Theory of Planned Behaviour [15]), current levels of physical, intentions to engage in physical activity, inhaler use, and finally reactions to specific vignettes relating to projected inhaler use and physical activity decisions. For the purposes of the current paper, only the vignette-related responses will be discussed. It is intended that the remaining wIME findings will be published elsewhere in the future.

The vignettes were balanced in terms of gender, age of character within vignette (all fell within 12-18 year age range), asthma severity, asthma control, level of physical exertion required for the activity and inhaler use. Sixteen vignettes were created in total: eight for pre-intervention and eight for post-intervention, with eight female and eight male vignettes. Pre- and post- intervention, participants responded to four vignettes. Male participants read versions of the vignettes with a named male character and females received vignette versions with a named female character in order to increase the potential for vicarious learning, in line with e-learning and artificial intelligence 'co-learner' theories [16]. The vignettes were developed in line with empirical guidance on effective vignette development $[17,18]$ and were piloted and fed-back on by the consultative user group prior to their use in the evaluation. Vignettes were all of approximately equal length (approx 150 words), with all provided information being directly relevant to the study's focus, with consistent volume, type and order of presented information being present across vignettes. Each vignette asked participants to imagine that they were the character and say whether they would take their inhaler (inhaler use simulation; two questions which were summed and an average score was calculated) and whether they would engage in physical activity (physical activity simulation; two questions which were summed and an average score was calculated), and how difficult it was to make these judgements. All of the vignette based questions were answered on a seven point likert-style scale, with 1 indicating a less positive response and 7 indicatng a more positive response.

A topic guide was used to help structure and focus the interviews and focus groups. However, these were mainly participant led discussions and the discussions were therefore not highly structured. Framework analysis was used, following Published guidance [19] to analyse the data. Framework analysis is a systematic form of thematic analysis which uses clear steps for the analysis process and produces highly structured, summarised data. It has been successfully used in policy research for 25 years and is increasingly being used to analyse qualitative data collected in applied health research [19]. Key themes and sub-themes were identified both within the individual interviews and focus groups and across the whole data set, providing a descriptive overview of the whole data set. Standardised consent forms and information sheets were used in all of the evaluation processes to ensure informed consent, and interviews and focus groups were audio-recorded using a digital audio recording device. 


\section{$2.4 \quad$ Procedure}

The wIME. Participants were recruited online, via a dedicated link to the baseline questionnaire (pre-intervention). After reading the information sheet and consenting to participation in the study, participants completed the questionnaire which assessed their current knowledge a about asthma, knowledge about inhaler use, and intention to increase physical activity. Following on from the questionnaire, the participants read four vignettes which described a young person with asthma who was engaging in a physically active task and answered questions relating to inhaler use and physical activity engagement based on the vignettes. Upon completion, participants were directed to a debriefing page where they were asked for their contact email address in order for them to be invited to participate in the second part of the study and, by means of incentive, were entered into a competition to win an iPod if they chose to take part in the second stage. After approximately two weeks, participants were emailed and invited to take part in the second stage of the study. They were provided with a link to take part in either the control condition (no animation) or the experimental condition (animation). Participants in the control condition completed the same questionnaire as in the first stage, and then were presented with four new vignettes that were designed to be balanced with/equivalent to the four that they had previously read. They again answered questions on these. They were then debriefed and were offered the opportunity to view the animation (for fairness). Participants in the experimental condition first viewed the animation and then completed the questionnaire and vignettes, and were then debriefed.

One-to-one in-depth qualitative interviews and focus groups. Participants read a standardised information sheet and completed a consent form prior to the session beginning, and all sessions took part in a suitable, private room to avoid distractions. Participants were verbally informed that the researchers were interested in their assessments of and interpretations of the animation that had been developed. The purpose of the animation was not disclosed until after the procedure (during debriefing) to avoid confounding the interpretations or biasing the participants' viewpoints. Participants were asked to view/use the animation and were told that they could work through it as many times as they wanted and that they could either comment on the animation as they were using it (i.e., 'think aloud' techniques, commonly used within clinical evaluations and for usability testing [20]; in line with methods previously used in similar contexts [1]). Following the viewing of the animation, the interviews/focus groups were carried out. These began by asking the participant(s) to explain what the animation was trying to convey, and the participants' interpretations were then used to structure the subsequent discussion(s). Following the interviews and focus groups, participants were fully debriefed.

\section{$3 \quad$ Results}

\subsection{Results of the wIME}

To test the Hypothesis that the animation would increase people's simulated intentions to engage in physical activity, a $2 \times 2$ mixed factorial ANOVA was conducted, with the between groups independent variable being the control vs animation condition and the 
within groups independent variable being the pre/post intervention. The dependent variable was participants' ratings for simulated intentions to engage in physical activity. The results are shown in Table 1 . A main effect of time was found, $F(1,19)=23.45$, $\mathrm{p}<0.001, \mathrm{n}^{2} \mathrm{p}=0.552$, indicating that when completing the questionnaire at time point two (post-intervention), participants had a greater intention to engage in physical activity (mean 3.95) than when they completed the questionnaire at time point one (pre-intervention; mean 2.89). No main effect of condition (control vs animation) or interaction was present and so this change cannot be attributed to the animation.

Table 1. Descriptive statistics for ratings of simulated intentions to engage in physical activity across the control/animation conditions and the pre/post intervention time points

\begin{tabular}{cllll}
\hline & & N & Mean & SD \\
\hline \multirow{2}{*}{$\begin{array}{c}\text { Pre- } \\
\text { intervention }\end{array}$} & Control & 12 & 2.94 & 0.93 \\
\cline { 2 - 5 } & Animation & 9 & 2.81 & 0.75 \\
\cline { 2 - 5 } Post- & Total & 21 & 2.89 & 0.84 \\
\hline \multirow{2}{*}{$\begin{array}{c}\text { Control } \\
\text { Intervention }\end{array}$} & 12 & 3.93 & 0.31 \\
\cline { 2 - 5 } & Animation & 9 & 3.99 & 0.04 \\
\hline & Total & 21 & 3.95 & 0.23 \\
\hline
\end{tabular}

To test the hypothesis that the animation would increase people's intentions to engage in safe/proper inhaler use, a $2 \times 2$ mixed factorial ANOVA was conducted, with the between groups independent variable being the control vs animation condition and the within groups independent variable being the pre/post intervention. The dependent variable was participants' ratings for intentions to engage in safe/proper inhaler use. The results are shown in Table 2. A main effect of time was found, $F(1,18)=81.74$, $\mathrm{p}<0.001, \mathrm{n} 2 \mathrm{p}=0.820$, indicating that when completing the questionnaire at time point two (post-intervention), participants had a lower intention to engage in safe/correct inhaler use (mean 3.93) than when they completed the questionnaire at time point one (pre-intervention; mean 4.66). No main effect of condition (control vs animation) or interaction was present and so this change, again, cannot be attributed to the animation.

Table 2. Descriptive statistics for ratings of simulated inhaler use across the control/animation conditions and the pre/post intervention time points

\begin{tabular}{cllll}
\hline & & N & Mean & SD \\
\hline \multirow{2}{*}{$\begin{array}{c}\text { Pre- } \\
\text { intervention }\end{array}$} & Control & 13 & 4.69 & 0.26 \\
\cline { 2 - 5 } & Animation & 7 & 4.61 & 0.21 \\
\cline { 2 - 5 } & Total & 20 & 4.66 & 0.24 \\
\hline \multirow{2}{*}{$\begin{array}{c}\text { Post- } \\
\text { Intervention }\end{array}$} & Control & 13 & 4.00 & $<0.01$ \\
\cline { 2 - 5 } & Animation & 7 & 3.79 & 0.57 \\
\cline { 2 - 5 } & Total & 20 & 3.93 & 0.34 \\
\hline
\end{tabular}


Finally decision difficulty relating to activity intentions and inhaler use intentions was investigated. In order to assess whether decision difficulty increased overall between the pre/post intervention conditions and across the control and animation conditions, a 2x2 mixed factorial ANOVA was carried out. No significant main effects or interactions $(\mathrm{p}>0.05)$ were found, indicating that rating decision difficulty was not different across the pre/post intervention time points or across the control/animation conditions. These findings are illustrated in Table 3.

Table 2. Descriptive statistics for ratings of decision making difficulty across the control/animation conditions and the pre/post intervention time points

\begin{tabular}{cllll}
\hline & & N & Mean & SD \\
\hline \multirow{3}{*}{$\begin{array}{c}\text { Pre- } \\
\text { intervention }\end{array}$} & Control & 11 & 5.11 & 0.21 \\
\cline { 2 - 5 } & Animation & 7 & 5.36 & 0.64 \\
\cline { 2 - 5 } & Total & 18 & 5.21 & 0.43 \\
\hline \multirow{2}{*}{$\begin{array}{c}\text { Post- } \\
\text { Intervention }\end{array}$} & Control & 11 & 5.14 & 0.26 \\
\cline { 2 - 5 } & Animation & 7 & 4.86 & 0.38 \\
\cline { 2 - 5 } & Total & 18 & 5.03 & 0.33 \\
\hline
\end{tabular}

A Pearson's correlation was carried out to identify whether there were any relationships between decision difficulty, inhaler intentions and physical activity intentions between the control and animation groups (post-intervention ratings only). None of the relationships were statistically significant ( $\mathrm{p}>0.05)$. However, the relationship between decision difficulty and physical activity simulation ratings was approaching significance, $\mathrm{r}=-0.445, \mathrm{p}=0.06, \mathrm{r} 2=0.198$ ( $19.8 \%$ shared variance). This relationship is illustrated in Figure 1; as shown, it appears that as decision difficulty increases, the intention to take part in physical activities decreases.

\subsection{Results of the Qualitative Evaluation}

Seven key themes emerged from the data: asthma and personal experiences; asthma and medication; physical activity at school; physical activity outside school; the animation; the support of friends and family; and translating their knowledge into practice. Of particular interest in the context of the current paper are the themes: 'asthma and medication', 'the animation' and 'translating knowledge into practice.' These will be discussed further, with a fuller and more detailed description of the rest of the themes being planned for a future publication. In relation to 'asthma and medication', participants discussed their current understandings of their medication and how it works, and related this to their change in knowledge following viewing the animation. This was largely a positive change, for example: "P: And especially the bit where it showed the little capillaries inside the canister... cause I think for children... to see it, so I think that actually just puts it in their head that there is actually something in there... so I felt that was really good, especially for wee ones. Just cause... you know, cause he was quite young when he got it, so I think that was quite... maybe it was 


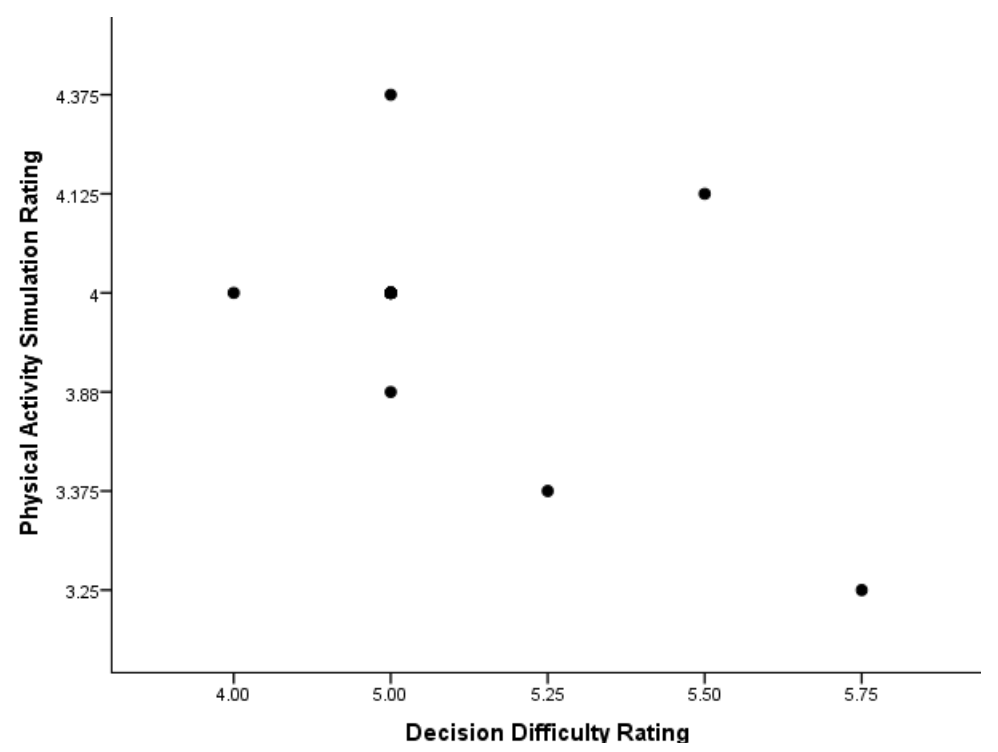

Fig. 1. Relationship between decision difficulty and physical activity simulation ratings for time point 2 (post-intervention)

more age for maybe seven or eight I think or even smaller it could be even more simplified, but I think it's very good."

In relation to 'the animation', participants responded to and directly discussed the animation and their attitudes towards it. Again, his was largely positive, with comments relating to the animation look ("P: When I saw the one with AJ, he did the one activity that I also do"), content ("INT: Was it too much information for them? P: I don't think for a 12 year old, it's quite basic, it's quite basic for Sarah's age and maybe to 18 , but for kids and stuff I think it's good. It was explained, it simplified it down which was good and it showed you how... would work"), and purpose ("INT: So how helpful did you find, in your own words, how helpful did you find the animation? P: I found it quite helpful because it did explain to you well how you can do things if you have asthma, and even if you need to take your inhaler during it and you can still keep going") being viewed as acceptable and useful.

In relation to 'translating knowledge into practice', participants discussed the ways that their new knowledge about their asthma (through watching the animation) may influence their future participation in physical activity, expressing initial difficulties/concerns with this: "INT: So would you consider being more physically active, but I guess you have to talk with your GP or your health professionals and things before that, but your view of physical activity and asthma, d'you find it difficult? P: Yeah I find it quite difficult to do stuff like that but watching that, telling you what to do and stuff before sport and stuff"; and then later expressing increased motivation to engage in physical activity, "INT: Okay. And after viewing the animation, d'you think you're more motivated to be more physically active? P: Definitely." 


\section{Discussion}

The present research aimed to identify whether a theory informed interactive animation could be used to increase intentions to engage in physical activity and improve knowledge about and intentions to use inhalers safely among young people with asthma and relevant others involved in their health care (i.e., parents, school staff, health professionals), and to identify whether the animation was acceptable to and meaningful to these groups. These aims were measured through an online evaluation (wIME), one-to-one in depth interviews and focus groups. The findings of the evaluation were mixed, and therefore indicates that further investigation is required.

The qualitative evaluations did indeed indicate that young people with asthma, parents, school staff and health professionals saw value in the animation, believing that it increased their knowledge about asthma; specifically, what happens in the lungs when a person is breathless due to asthma compared to when they are simply breathless due to being active, and increasing knowledge about inhaler use and purpose. Of particular positive note for the animation in terms of design and HCI, participants indicated that the animation's look was appealing, the level of choice in terms of characters and activities was advantageous to their immersion and experience, and that the level of information was about right (i.e., not too detailed and not too basic).

The qualitative findings are highly positive, and indicate that the animation may hold some promise. However, the quantitative findings are less indicative. Findings did indicate that the intention to engage in physical activity was greater at the second time-point (post-intervention questionnaire), but this increase was not statistically related to or accounted for by the animation itself. In addition, significant differences were found between the pre/post intervention ratings for participant's ratings of safe/proper inhaler use simulations. In this instance, participants' ratings actually decreased, indicating poorer intentions to use inhalers properly; and, again, this decrease was not associated with the animation itself. It can only be assumed that something outwith the study's measurements was influencing or mediating these intention ratings.

In order to further investigate and to clarify the discrepancy between the qualitative and quantitative findings, the research team plan to engage in a full trial to assess whether the apparent increased intentions, expressed in the qualitative evaluations, to engage in more physical activity will indeed translate to a change in behaviour. This follow on work will also include an assessment of the efficacy of including an associate individualised 'action plan' to help promote a concrete pathway to behaviour change following viewing the animation. This will be a key challenge, as it is well known within the psychological literature that even though someone may express the intention to change a behaviour, the actual behaviour change may not follow (i.e., the intentions-behaviour gap). 


\section{References}

1. Williams, B., Anderson, A., Barton, K., McGhee, J.: Can theory be embedded in visual interventions to promote self-management? A proposed model and worked example. International Journal of Nursing Studies 49(12), 1598-1609 (2012)

2. Williams, B., Cameron, L.: The potential and problems of the increasing use of images in health care interventions: Are we running before we can walk? Journal of Health Services Research \& Policy 14(4), 251-254 (2009)

3. Craig, P., Dieppe, P., MacIntyre, S., Michie, S., Nazareth, I., Petticrew, M.: Developing and evaluating complex interventions: new guidance. Medical Research Council, 1-39 (2008)

4. Murray, J., Williams, B., Hoskins, G., McGhee, J., Gauld, D., Brown, G.: Developing a theory-informed interactive animation to increase physical activity among young people with asthma. In: Shumaker, R. (ed.) VAMR 2013, Part II. LNCS, vol. 8022, pp. 60-65. Springer, Heidelberg (2013)

5. Anandan, C., Nurmatov, U., Schayck, O., Sheikh, A.: Is the prevalence of asthma declining? Systematic review of epidemiological studies. Allergy Asthma Proc. 65(2), 152-167 (2009)

6. Asher, M.I., Montefort, S., Bjorksten, B., Lai, C.K.W., Strachan, D.P., Weiland, S.K., et al.: Worldwide time trends in the prevalence of symptoms of asthma, allergic rhinoconjunctivitis, and eczema in childhood: ISAAC Phases One and Three repeat multicountry cross-sectional surveys. The Lancet 368(9537), 733-743 (2006)

7. Beasley, R.: The International Study of Asthma and Allergies in Childhood (ISAAC) Steering Committee. Worldwide variation in prevalence of symptoms of asthma, allergic rhinoconjunctivitis, and atopic eczema: ISAAC. The Lancet 351(9111), 1225-1232 (1998)

8. Dollman, J., Norton, K., Norton, L., Cleland, V.: Evidence for secular trends in children's physical activity behaviour. Commentary, 892-897 (2005)

9. Glazebrook, C., McPherson, A.C., Macdonald, I.A.S., J. A, Ramsay, C., Newbould, R., Smyth, A.: Asthma as a barrier to children's physical activity: implications for body mass index and mental health. Pediatrics 118(6), 2443-2449 (2006)

10. van Veldhoven, N.H.M.J., Vermeer, A., Bogaard, J.M., Hessels, M.G.P., Wijnroks, L., Colland, V.T., et al.: Children with asthma and physical exercise: effects of an exercise programme. Clinical Rehabilitation 15, 360-370 (2001)

11. Welsh, L., Kemp, J.G., Roberts, R.G.D.: Effects of physical conditioning on children and adolescents with asthma. Sports Medicine 35(2), 127-141 (2005)

12. Bonetti, D., Eccles, M., Johnston, M., Steen, N., Grimshaw, J., Baker, R., et al.: Guiding the design and selection of interventions to influence the implementation of evidencebased practice: An experimental simulation of a complex intervention trial. Social Science and Medicine 60, 2135-2147 (2005)

13. Hrisos, S., Eccles, M., Johnston, M.: An intervention modelling experiment to change GPs' intentions to implement evidence-based practice. BMC Health Services Research 8(10) (2008)

14. Yeatts, K., Sotir, M., Music, S., Herget, C.: Health consequences for children with undiagnosed asthma-like symptoms. Arch. Pediatr. Adolesc. Med. 157, 540-544 (2003)

15. Ajzen, I.: The theory of planned behaviour. Organisational Behavior and Decision Processes 50, 179-211 (1991)

16. Lee, J.R., Nass, C., Brave, S.B., Morishima, Y., Nakajima, H., Yamada, R.: The case for caring colearners: The effects of a computer-mediated colearner agent on trust and learning. Journal of Communication 57, 183-204 (2007) 
17. Ashill, N.J., Yavas, U.: Vignette development: An exposition and illustration. Innovative Marketing 2(1), 28-36 (2006)

18. Heverly, M.A., Fitt, D.X., Newman, F.L.: Constructing case vignettes for evaluating clinical judgment: An empirical model. Evaluation and Program Planning 7, 45-55 (1984)

19. Gale, N.K., Heath, G., Cameron, E., Rashid, S., Redwood, S.: Using the framework method for the analysis of qualitative data in multidisciplinary health research. BMC Medical Research Methodology 13, 117 (2013)

20. Jaspers, M.W.M.: A comparison of usability methods for testing interactive health technologies: Methodological aspects and empirical evidence. International Journal of Medical Informatics 5, 340-353 (2009) 